\title{
STATIC NETWORK CODE DGPS POSITIONING VS. CARRIER PHASE SINGLE BASELINE SOLUTIONS FOR SHORT OBSERVATION TIME AND MEDIUM-LONG DISTANCES
}

\author{
M. Bakuła \\ University of Warmia and Mazury in Olsztyn, \\ Chair of Satellite Geodesy and Navigation, Poland. \\ E-mail: mbakula@uni.olsztyn.pl
}

\begin{abstract}
GPS land surveys are usually based on the results of processing GPS carrier phase data. Code or pseudorange observations due to considerations of accuracy requirements and robustness are preferred in navigation and some GIS applications. Generally, the accuracy of that positioning is in the range of about 1-2 meters or so, on average. But the main problem in code GPS positioning is to know how to estimate the real accuracy of DGPS positions. It is not such an easy process in code positioning when one reference station is used. In most commercial software, there are no values of accuracy but only positions are presented. DGPS positions without estimated errors cannot be used for surveying tasks and for most GIS applications due to the fact that every point has to be have accuracy determined. However, when we used static GPS positioning, it is well known that the accuracy is determined, both during baseline processing and next by the adjustment of a GPS network. These steps of validation with redundancy in classical static phase baseline solutions allow wide use of static or rapid static methods in the main land surveying tasks. Although these control steps are commonly used in many major surveying and engineering tasks, they are not always effective in terms of short-observation-time sessions. This paper presents a new network DGPS approach of positioning with the use of at least three reference stations. The approach concerns also valid accuracy estimation based on variance-covariance (VC) matrix in the least-squares (LS) calculations. The presented network DGPS approach has the ability of reliable accuracy estimation. Finally, network DGPS positioning is compared with static baselines solutions where five-min sessions were taken into consideration for two different rover stations. It was shown that in a short observation time of GPS positioning, code network DGPS results can give even centimetre accuracy and can be more reliable than static relative phase positioning where gross errors often happen.
\end{abstract}

\section{INTRODUCTION}

There are many different needs in practice for surveying, engineering or navigation tasks in terms of accuracy of GNSS (Global Navigation Satellite Systems) positioning. For instance, there are centimetre needs for control points or boundary lines, and decimetre for boundaries of lakes, ditches or so. In GPS positioning, however, it is not so easy to obtain reliable accuracy with decimetre positioning. If traditional available GPS methods of positioning are used, there are methods based on carrier phase with centimetre accuracy or based on code/pseudorange observations used rather in navigation or in some Geographic Information 
Systems (GIS) where an accuracy of about 1-2 meters is expected. The 1-2 meter accuracy is sufficient enough, for instance, in fleet management but not enough in present demands for e.g. precise agriculture or some GIS tasks. For the present demands for GIS database, quick GNSS positioning with a reliable accuracy of $20-30 \mathrm{~cm}$ is extremely very welcome. For precise GPS positioning, RTK technology can be used but that approach has some strong demands in terms of very good GPS observational conditions. Lack of good satellite configuration and some obstructions causing very long ambiguity resolution times or even not allow obtaining successfully solutions. Sometimes, it is quite easy to meet gross errors in final coordinates in relative phase positioning, both in real-time (Bakuła and Oszczak, 2006; Bakuła et al., 2006) or in post-processing when the time of observation is not so long (Bakuła, 2006b). It is well known that centimetre accuracy can be obtained when using GPS phase data. It is also well known that in the phase GPS methods, a key issue is a proper solution of the so-called integer ambiguities. It is the key to high accuracy positioning for a single baseline. Because the phase ambiguities are integer values, the most accurate phase GPS technique resolve integer ambiguities, resulting in relative positioning accuracies at the $\mathrm{cm}$ level. Carrier phase ambiguity resolution is limited in longer baselines by measurement errors that are not removed in the double-referencing process. These errors are grouped into spatially correlated errors (atmospheric and satellite position errors) and uncorrelated errors (receiver noise and multipath). In order to reduce spatially correlated errors, some network approaches have been elaborated during the last decade (Wübbena et al., 1996; Wanninger, 1995; Raquet, 1997; Landau et al., 2002; Lachapelle and Alves, 2002; Grejner-Brzezińska et al., 2005). One of the most important advantages for multi-reference station network users, as compared to single baseline users, is modelling of the distance-dependent or spatially correlated errors such as ionospheric, tropospheric and satellite effects. By combining observations from a number of reference stations, the effect of the above mentioned distance-dependent errors can be reduced, through various techniques. Although the presented network methods seem much more reliable than classical positioning we should note that the final results depend not only on the quality of base data, but also on the rover observations. It means that the quality of rover GPS data is still essential in baseline solutions.

The traditional DGPS positioning approach was studied in many papers worldwide where practical tests show that the accuracy of code differential positioning is within 1-2 $\mathrm{m}$ and sometimes even more. Due to the wide use of network stations as future solutions in GNSS approaches, this paper presents the code network approach versus traditional static baseline solutions in short observation periods (5-min sessions). The approach is based on linear interpolation of pseudorange corrections (PRC) and, additionally, a smoothing process of determined final positions is implemented. Additionally, in the iterative LS solution a weight matrix is used for valid and reliable accuracy estimation of determined positions.

\section{CODE NETWORK DGPS POSITIONING}

In traditional DGPS positioning, the reference station calculates pseudorange corrections that are applied to the remote receiver. DGPS coordinates can be determined with the use of LS algorithms. In order to employ the LS method, both the functional and stochastic models of the GPS measurements should be defined. The functional model describes the mathematical relations between pseudorange measurements and the unknown parameters such as the coordinates in the global geodetic system. In dependence on a method of GPS positioning we can find in the literature [for example, in such textbooks as Hofmann - Wellenhof et al, 1997; Kleusberg and Teunissen (eds), 1996; Leick, 1995; Parkinson and Spilker (eds), 1996; Strang and Borre, 1997; Misra and Enge, 2001] many different approaches of functional models. 
If we want to determine the coordinates of an unknown point in the global geocentric coordinate system, we must perform linearization of the observed equation. Let us denote the vector of observation by $\mathrm{L}$, the vector of unknowns by $\mathrm{X}$ and the design matrix by $\mathrm{A}$. Then, employing the LS method, the estimator of the unknown vector $\hat{X}$ equals $\hat{\mathrm{X}}=\left(\mathrm{A}^{\mathrm{T}} \mathrm{PA}\right)^{-1} \mathrm{~A}^{\mathrm{T}} \mathrm{PL}$, where: $\mathrm{A}$-design matrix, $\mathrm{P}$ - is a weight matrix.

At the beginning of the iterative procedure LS adjustment, we use the unity matrix, but later we can use the weights different for every satellite, calculated according to (Bakuła, 2006a):

$$
p_{n}=\frac{1}{\left[P R C_{R E F V}^{n}(t)-P R C_{P K T 1}^{n}(t)\right]^{2}}
$$

where: $P R C_{R E F V}^{\text {satn }}(t)$ - represents pseudorange corrections for the virtual reference station but $P R C_{P K T 1}^{n}(t)$ is for the rover (unknown) station.

In surveying, it is well known that the values of $\hat{\mathrm{X}}$ depend on functional and stochastic models. Both of them have an influence on the final results. In the presented network DGPS positioning we applied the weight matrix, following the assumption that the accuracy of pseudorange measurements obtained from particular satellites is not the same. The different weights for each satellite (Eq. 1) reflect the linear behaviour of pseudorange corrections in network code DGPS positioning. The matrices $\mathrm{L}$ and $\mathrm{P}$, in the iterative LS adjustment method, can reduce time-spatial errors, pseudorange noise and, especially, the weight matrix eliminates gross errors taking place at the reference stations and the rover station. In order to reduce distance-dependent errors, with at least three reference stations in the vicinity of an unknown control point, virtual reference pseudorange corrections are calculated using linear interpolation.

When the iterative process of the LS solution is finished, the statistics for the adjusted estimated unknown coordinates should be computed. The first item to be computed is the variance factor $\sigma_{0}$. The second, the standard deviations for the estimated station coordinates should be computed. They are obtained from variance-covariance (VC) matrix of the adjusted parameters, $\Sigma_{\mathrm{x}}=\sigma_{0}^{2}\left(\mathrm{~A}^{\mathrm{T}} \mathrm{PA}\right)^{-1}, \quad \sigma_{0}=\left[\left(\mathrm{V}^{\mathrm{T}} \mathrm{PV}\right) /(\mathrm{m}-\mathrm{n})\right]^{1 / 2}$, where $m-n$ is the total redundancy of the system.

\section{RELATIVE PHASE POSITIONING}

Classical static GPS positioning is based on continuous simultaneous observations of two or more receivers for approximately half an hour or more, depending on the measured lengths of baselines. The baseline components are determined from carrier phase data. The high precision of the determination is mainly derived from the change in satellite geometry over the time session observed. With dual-frequency data, solutions can be obtained for different frequencies and frequency combinations such as L1, L2, Lw (wide lane), Lc (ionosphere free), Ln (narrow lane). Dual-frequencies data allow the positioning of so-called "short static" or "rapid/fast static". Short static is a special type of the static method where baselines are calculated by efficient phase ambiguity resolutions. In static positioning, an observational time and accuracy are a function of baseline length and number of visible satellites. In short static, it is recommended that baseline lengths should be kept to a minimum (Ashtech and Spectra Precision Terrasat, 1998).

The ambiguity resolution process relates to both two receivers and two satellites. It is a process of resolving the unknown cycle ambiguities of double difference carrier phase data as an integer. There is no time dependency as long as no interruption appears during GPS measurements. When the ambiguity is determined as an integer value, it is said that the 
process is resolved or fixed. The ambiguity fixing process strengthens the baseline solution. The use of double differences instead of single differences is much more effective due to the elimination of clock terms. There are many models in the literature dealing with this challenge that differ greatly in complexity and diversity. The general model of ambiguity estimation can be presented in linear observation equations (Teunissen at al., 1997)

$$
y=A a+B b+e
$$

where:

$y$ - is the vector of observed minus computed measurements,

$a$-is the vector of unknown integer ambiguities,

$b$ - is the vector of increments of the unknown baseline components,

$A, B$-are the corresponding design matrices for ambiguity terms and baseline components, respectively,

$e-$ is the vector of measurement noise and unmodelled errors.

The data vector consists of the observed minus computed phase and/or pseudorange observations. The elements of the vector $a$ are the double reference carrier phase ambiguities expressed in units of cycles and they are known to be integers except ionosphere-free combinations of observables. The elements of the vector $b$ consist of the remaining unknown parameters, for instance, baseline components and atmospheric delay parameters. They are known to be real values.

The ambiguity resolution is not an easy process due to their integer values. It becomes more difficult when the length of the baseline is long. Considering a short baseline, i.e. up to $20 \mathrm{~km}$, the model of double difference may be simplified, (Hofmann-Wellenhof et al., 1997)

$$
\lambda \Delta \nabla \phi_{A, B}^{j, k}(t)=\rho_{A, B}^{j, k}(t)+\lambda N_{A, B}^{j, k}+\text { noise }
$$

The above equation can be written with the assumption that the effect of the ionosphere, troposphere and others may be neglected, in general. But any residuals from these neglected terms will affect the ambiguity resolution process and degrade coordinates of the baseline. If the noise values are close, or even larger, than half of a phase cycle, valid ambiguity estimation is rather impossible due to bias errors. And, therefore, Eq. 3 is only valid for short baselines. For longer distances, the remaining errors (which are not cancelled in double differencing techniques) must be taken into consideration. Thus, network approaches are an alternative for GNSS positioning.

\section{NUMERICAL EXAMPLE}

The GPS data used in this chapter were collected at five known permanent reference stations of the ASG-PL (i.e. Polish Active Geodetic Network) network (Fig. 1): KLOB in Kłobuck, LELO in Lelów, ZYWI in Żywiec, KATO in Katowice and TARG in Tarnowskie Góry, Poland. The reference stations are equipped with Ashtech $\mu$ Z-CGRS (Continuous Geodetic Reference Station) receivers and ASH701945C_M SNOW antennas. The GPS observations were gathered on 17 April 2005, for one hour - from 13:00 to 14.00 UTC; 5-second intervals of data recording and the values of PDOP were between five and seven. Six satellites were used in the calculations, the same for both static baseline solutions and code network differential GPS. In the calculations presented in this paper, the stations TARG and KATO were treated as unknown, whereas the other three: KLOB, LELO and ZYWI were treated as reference stations. 
GPS positioning was carried out according to the schedule:

I) short 5-min sessions of static positioning for the baselines:

- KLOB-TARG (51 km), results in Figs: 2a, 3a, 4a;

- LELO-TARG $(60 \mathrm{~km})$, results in Figs: 2b, 3b, 4b;

- ZYWI-TARG $(89 \mathrm{~km})$, results in Figs: 2c, 3c, 4c;

- KLOB-KATO (73 km), results in Figs: 2d, 3d, 4d;

- LELO-KATO (64 km), results in Figs: 2e, 3e, 4e;

- ZYWI-KATO (64 km), results in Figs: 2f, 3f, 4f;

II) network DGPS positioning:

- for the rover station TARG, results in Fig. 5a;

- for the rover station KATO, results in Fig. 5b.

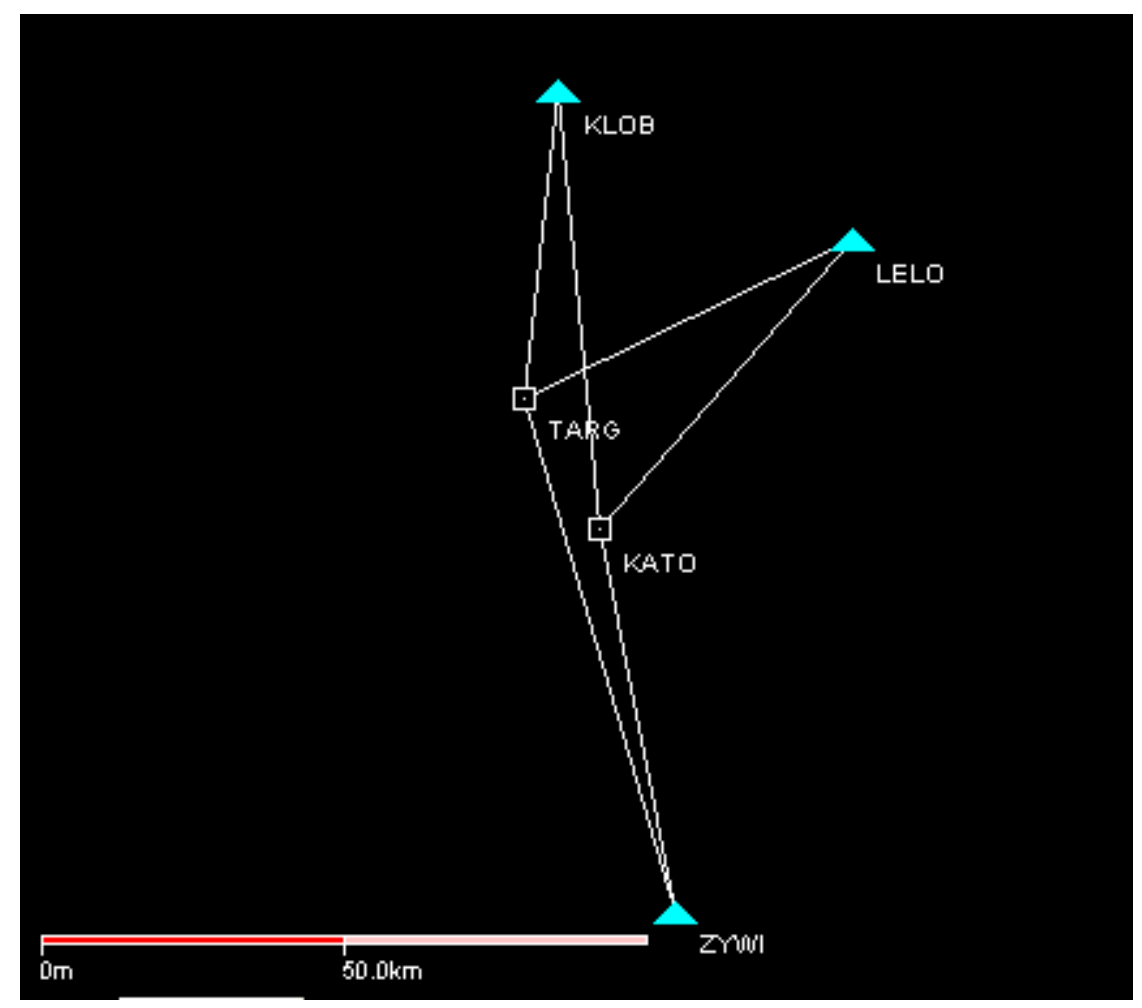

Fig. 1. Deployment of permanent reference stations of the ASG-PL network used in the tests 
In the presented static baseline calculations, the L1/L2 processing was implemented based on the Ashtech Office Suite (AOS) software. The AOS software automatically activates short static techniques if L1 and L2 processing is chosen and if dual-frequency data is available. The second frequency supports the ambiguity resolution. In that processing strategy, the AOS software at first determines the wide line ambiguity $(\mathrm{Lw})$ and afterwards fixes the narrow lane ambiguity (Ln) by processing the ionospherically free carrier observables Lc. After a successful fixing of Ln, the software outputs L1, L2, Lw, Ln and Lc fixed solutions (Ashtech and Spectra Precision Terrasat, 1998). In order to derive optimum phase ambiguities from the float solution, the AOS software applies a time-optimized search technique, which is defined on the sigma value of the determined float ambiguity, for the solutions with the smallest sum of squares residual error. Statistical testing is used to verify the correctness of the ambiguity resolution. First, a Chi-square is performed on the a posteriori variance of the residuals with a default of $95 \%$ minimum probability. Second, a Fisher test is performed with the ratios of the variances of the second to the best fitting solution with a reliability of $99.99 \%$. The ability for a fixed ambiguity solution is also limited by the required minimum ratio of variances for the Fisher test. Ashtech Office Suite uses a value of 2 by default.

Due to many possibilities of the AOS software, baseline calculations are divided into three main approaches:

I) calculations with the usage of L1, one frequency GPS data, (Fig. 2);

II) the wide line (Lw) approach of ambiguity resolution based on L1 and L2 GPS data, (Fig. 3);

III) the automatic solution made by the software where all possible linear combinations (L1, L2, Lw, Ln, Lc) are used in order to obtain the best results (Fig. 4).

According to the Ashetch company (1993), time observation span versus baseline length in rapid static for dual-frequency GPS receivers where all GPS data are taken advantage of is presented below:

\begin{tabular}{|c|c|c|c|c|c|c|}
\hline Line Length [km] & $1-5$ & 6 & 7 & 8 & 9 & 10 \\
\hline Session Time [min] & 10 & 12 & 14 & 16 & 18 & 20 \\
\hline
\end{tabular}

The presented observation times assume good satellite geometry ( $\mathrm{PDOP}<5$ ). It is noted by the company that successful processing may be achieved with shorter time periods and on longer lines but such results "cannot be guaranteed". It is also added by the company that rapid static observation can be considered to be successful when the ambiguity can be solved. 

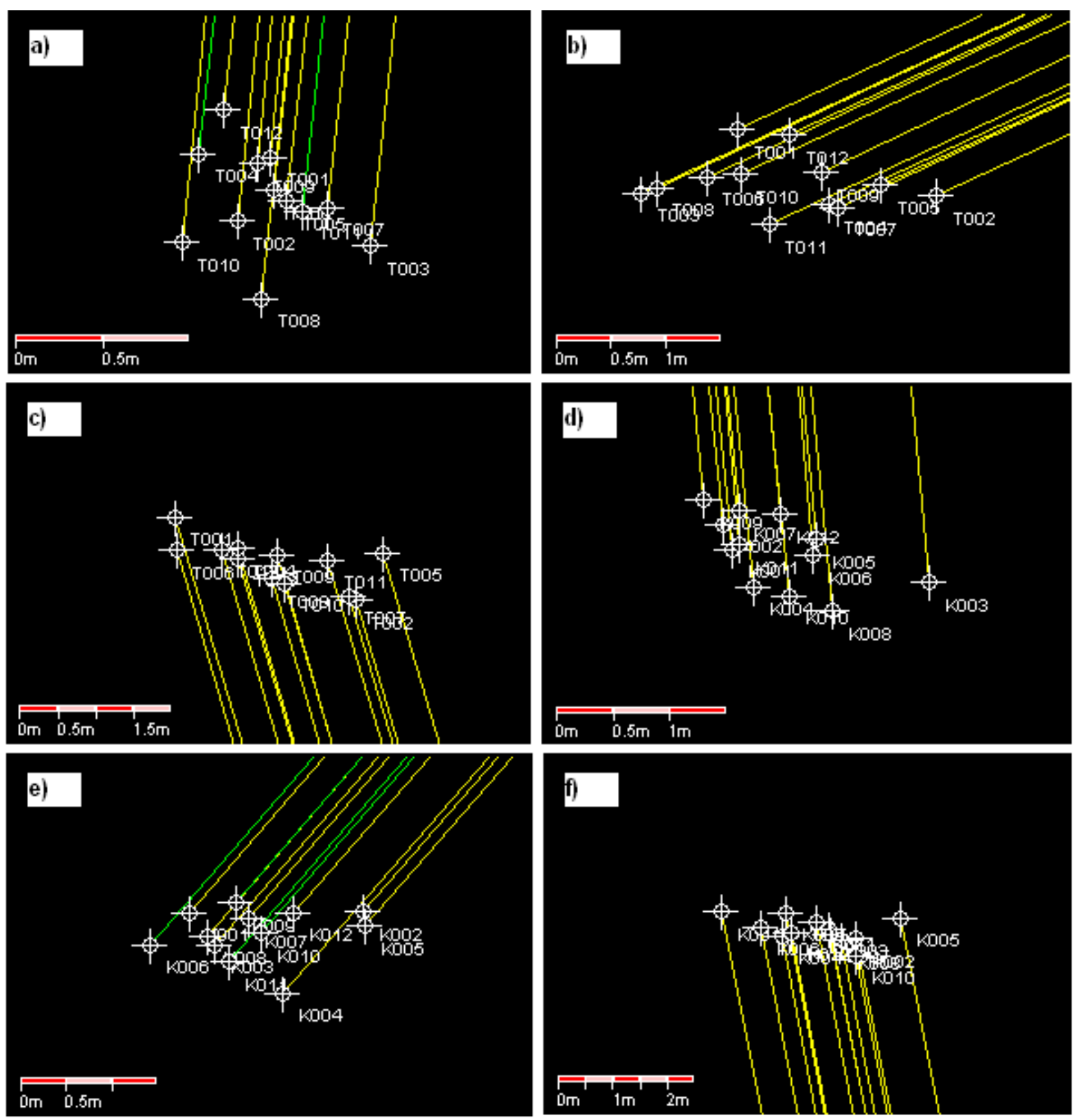

Fig. 2. Horizontal scatter of GPS solutions on L1 option for the baselines: a) KLOB-TARG, b) LELO-TARG, c) ZYWI-TARG, d) KLOB-KATO, e) LELO-KATO and f) ZYWI-KATO

In Fig. 2 there are solutions on L1 frequency. It can be seen that almost all solutions are partial (yellow colour), it means that the ambiguity solution process failed. In this situation, the results cannot be considered reliable and shouldn't be used for any mapping or surveying applications. The partial solutions were within 1-3 meters from the true coordinates. That accuracy is at the level of handheld GPS receivers, although the phase data were used to calculate the baselines. Only for the baselines KLOB-TARG and LELO-TARG were there two and four baselines fixed, respectively, though the final coordinates obtained from these solutions were in the range of 0.3- 1 meter different from the true coordinates. 

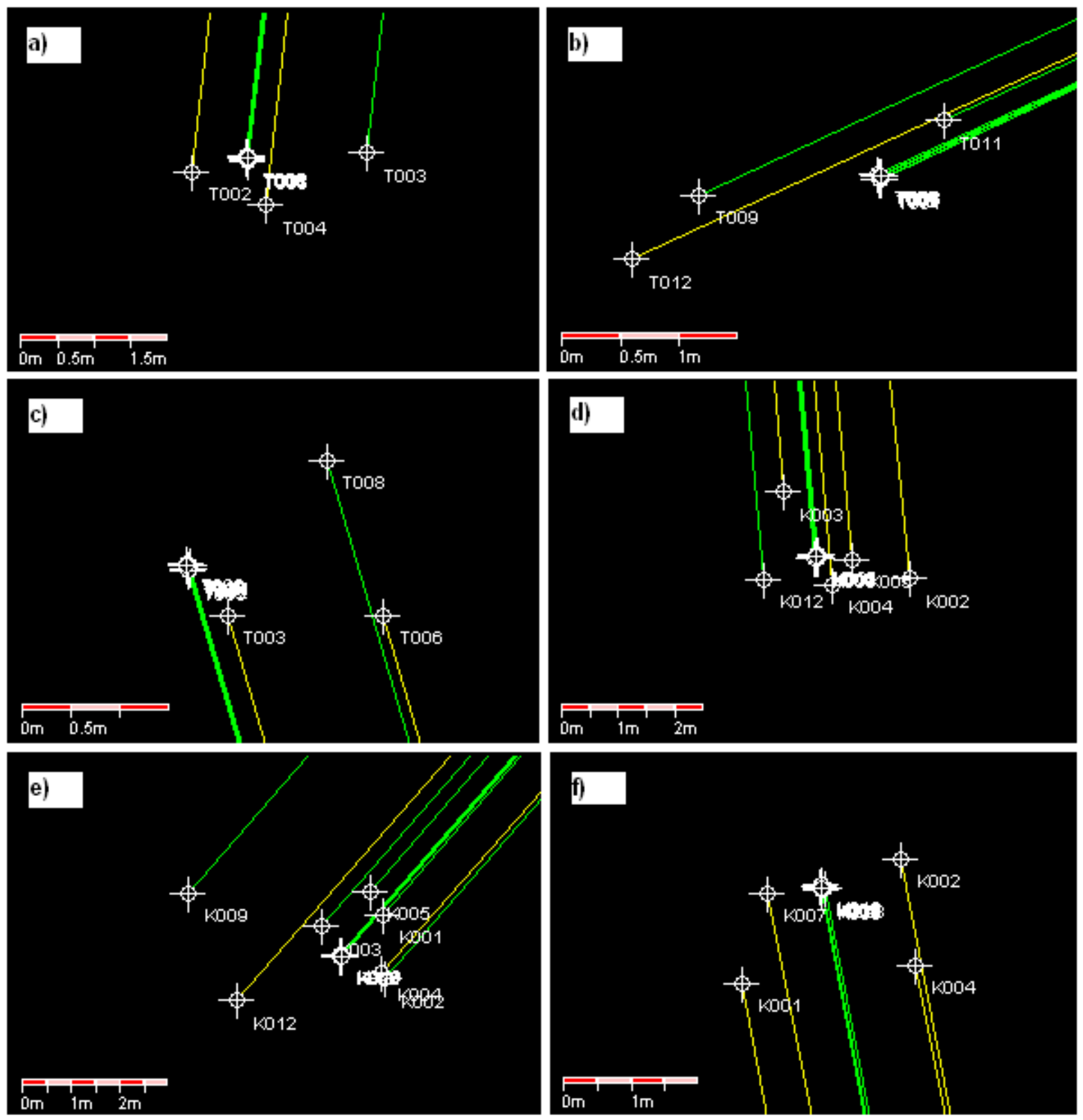

Fig. 3. Horizontal scatter of GPS solutions on Lw option for the baselines:

a) KLOB-TARG, b) LELO-TARG, c) ZYWI-TARG, d) KLOB-KATO, e) LELO-KATO and f) ZYWI-KATO

When the Lw approach of ambiguity was chosen (Fig. 3) much more fixed solutions could be seen than on the L1 option. For the shorter baseline KLOB-TARG (Fig. 3a), only two solutions were partial but others were fixed. The one (session T003) baseline was fixed but the coordinates were within $3 \mathrm{~m}$ from true coordinates. For the rest solutions of the baseline KLOB-TARG positions were within the radius of $5 \mathrm{~cm}$. For the baseline LELO-KATO (Fig. $3 b$ ), only one solution was partial but two fixed solutions had gross errors, the rest (nine solutions) were also within $5 \mathrm{~cm}$ from true coordinates. A similar situation was for the baseline ZYWI-TARG (Fig. 3c) where nine solutions were within the radius of $5 \mathrm{~cm}$, but one (session T008) had gross errors although the solution was fixed.

For the baselines KLOB-KATO (Fig. 3d), LELO-KATO (Fig. 3e) and ZYWI-KATO (Fig. 3f) the results were much worse, more partial solutions and more gross errors took place. Although it is clear that partial solutions could not be taken into consideration for further 
application in surveying, the fixed solutions with gross errors can happen quite often in that short observation time during the static Lw phase solutions.

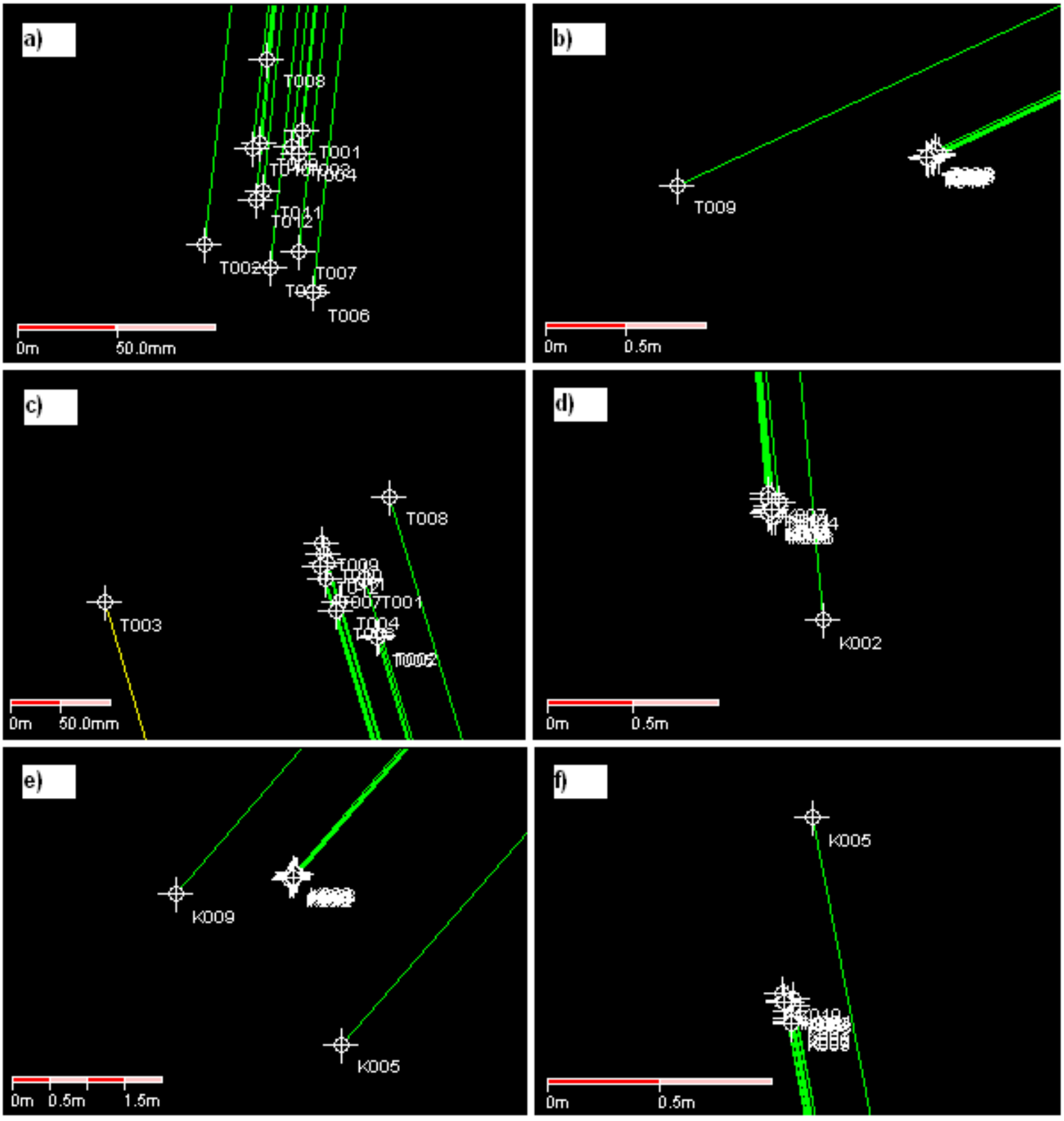

Fig. 4. Horizontal scatter of GPS solutions on L1/L2 option for the baselines:

a) KLOB-TARG, b) LELO-TARG, c) ZYWI-TARG, d) KLOB-KATO, e) LELO-KATO and f) ZYWI-KATO

In Fig. 4 the solutions are based on L1 and L2 data where some different linear combinations were taken advantage in baselines processing. In most baseline solutions the ambiguities were calculated as fixed (green colour) but one can see that baselines are scattered in the range of one meter or even more (e.g. Fig. 4e). It was caused by wrong ambiguities acceptance and therefore the final coordinates are biased.

At this moment it should be mentioned that 5 -min sessions are not recommended by the producers of GPS receivers or software. In this study, baselines from $51 \mathrm{~km}$ (KLOB-TARG) to $89 \mathrm{~km}$ (ZYWI-TARG) were used. This exceeded the distances for baselines commonly recommended by the manufacturers of GPS receivers and software. Nevertheless, the 5-min 
sessions were post-processed, in order to compare the results of static GPS baselines with the approach of network code DGPS positioning.

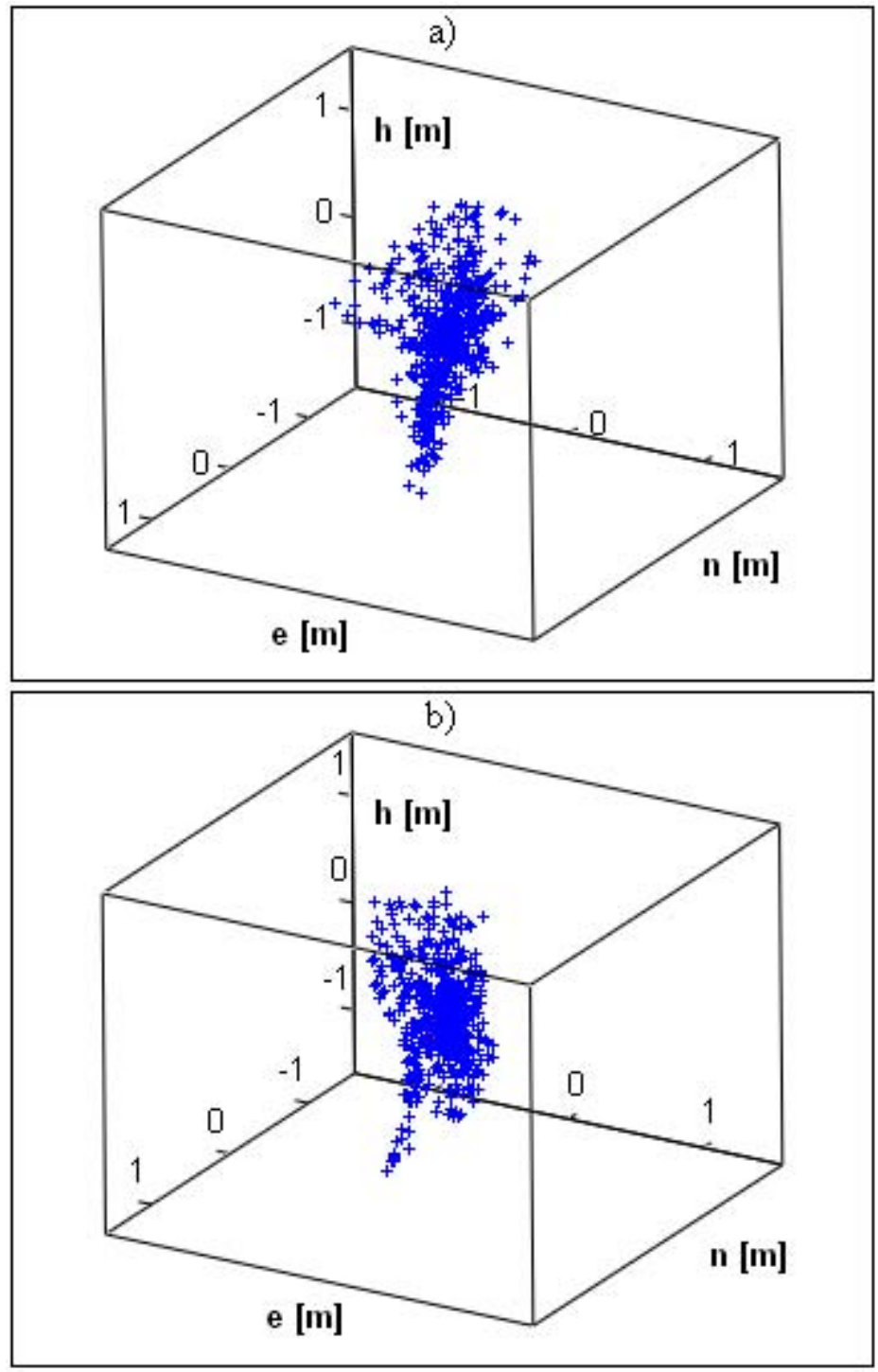

Fig. 5. 3D skater plot of positions in the network code DGPS positioning;

a) for the station TARG, b) for the station KATO

For both rover stations TARG and KATO "real" errors were calculated both in the global coordinate system $(\Delta \mathrm{X}, \Delta \mathrm{Y}, \Delta \mathrm{Z})$ as differences between the fixed coordinates and the coordinates obtained in successive epochs of differential code positioning and in a local topocentric coordinate system $(\Delta \mathrm{n}, \Delta \mathrm{e}, \Delta \mathrm{h})$. Fig. 5 presents $3 \mathrm{D}$ positions with the use of linear interpolation of pseudorange corrections based on three reference stations and different weights for a particular satellite but without smoothing process yet. Positions are presented in the local topocentric system $(\mathrm{n}, \mathrm{e}, \mathrm{h})$. It should be noted that every position was calculated independently. There are no relations between successive epochs.

Additionally, real errors were compared with values of standard deviations obtained from the variance-covariance matrix, of DGPS adjustment $\left(\mathrm{m}_{\mathrm{X}}, \mathrm{m}_{\mathrm{Y}}, \mathrm{m}_{\mathrm{Z}}\right)$. For the comparison of standard deviations from variance-covariance matrix and real errors obtained in each epoch, the so-called RMS difference $(\Delta r m s)$ term was introduced, calculated by the following equation: 


$$
\Delta r m s=\sqrt{\mathrm{m}_{\mathrm{X}}^{2}+\mathrm{m}_{\mathrm{Y}}^{2}+\mathrm{m}_{\mathrm{Z}}^{2}}-\sqrt{\Delta \mathrm{X}^{2}+\Delta \mathrm{Y}^{2}+\Delta \mathrm{Y}^{2}}
$$

The values of $(\Delta r m s)$ for two rover stations in network DGPS solutions are presented in Fig. 6. If the $\Delta r m s$ is around zero or larger, it is better. It shows that the accuracy from the VC matrix is reliable. If the $\Delta r m s$ is positive it means that estimated errors from VC matrix are larger than real errors. It should be said that this consistency is more visible for the variance factor around the unity.

a)

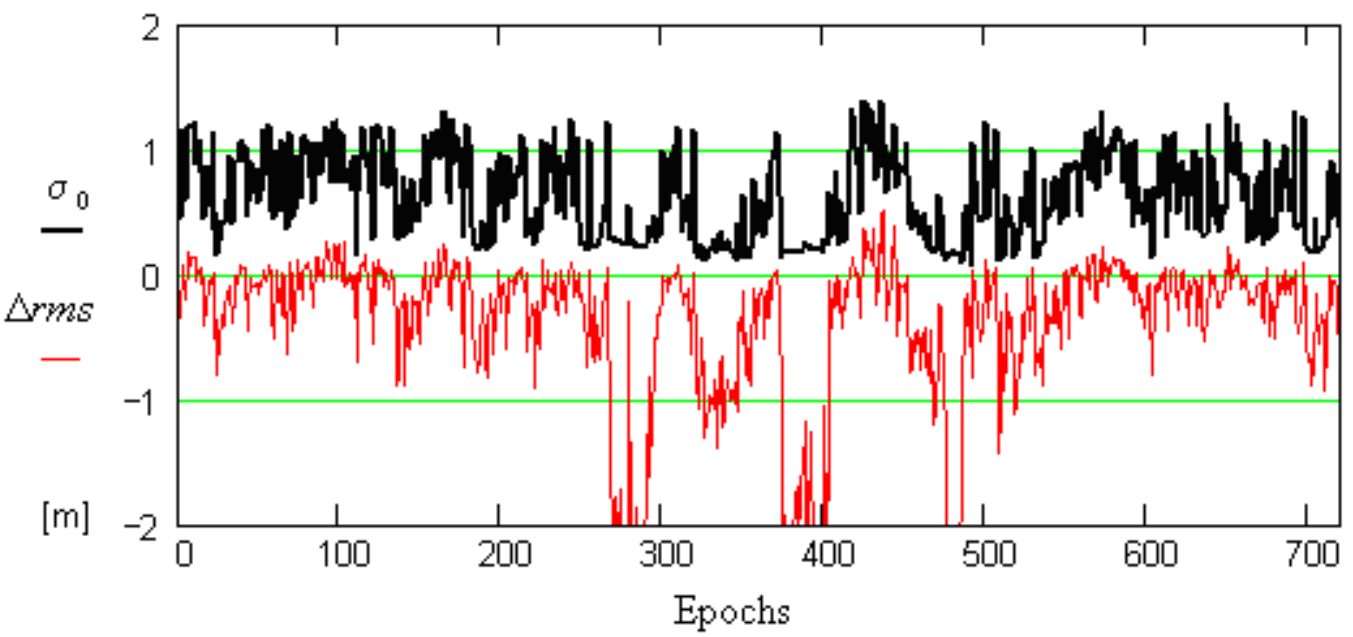

b)

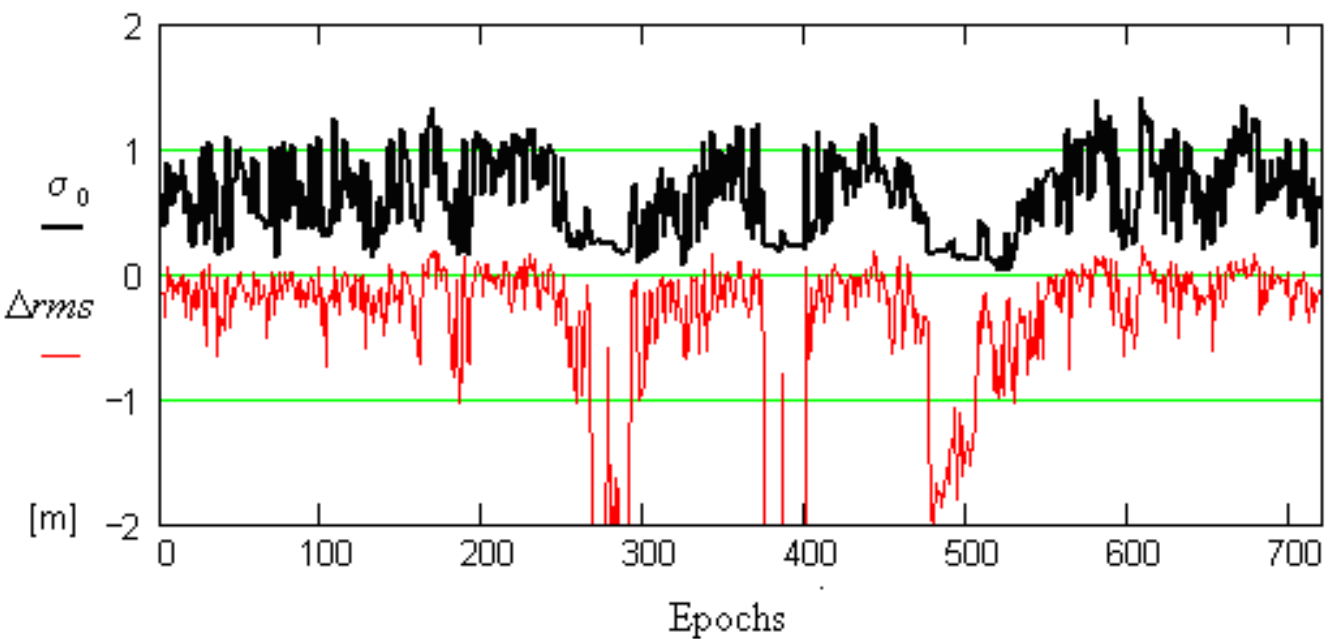

Fig. 6. Values of 3D RMS difference $(\Delta r m s)$ and values of the variance factor obtained in the network DGPS positioning: a) for the station TARG, b) for the station KATO

In Fig. 7 there are additionally smoothed network code positions, performed by the supsmooth function of Mathcad 11 software, used for the north, east and height components. We can see that for all data, the east and north components are much better in terms of accuracy than the height. Horizontal positions were in the range of up to $20 \mathrm{~cm}$ from the true coordinates whereas the height sometimes was even up to about one meter from the true position. Although a smoothing process was added, it is clear that that degradation of positions are when scale factor was far from unity. For these two rover stations, as much as 1440 positions were obtained and there were no epochs with bad accuracy estimation when the variance factor was in the range of 0.8-1.2. 
a)

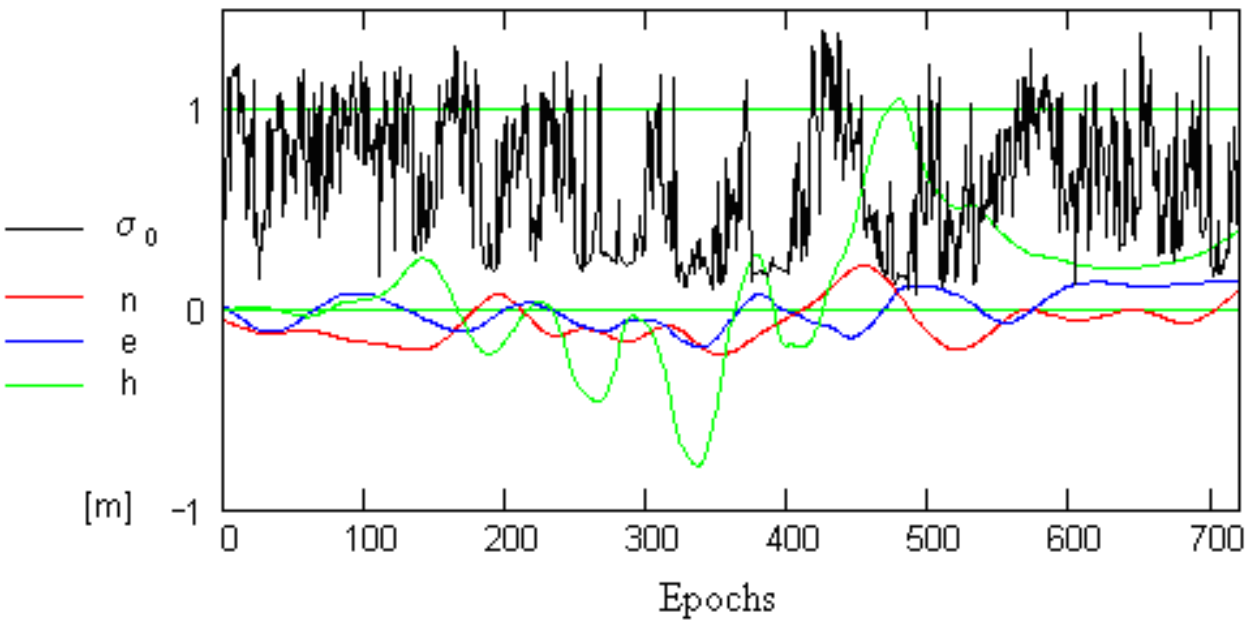

b)

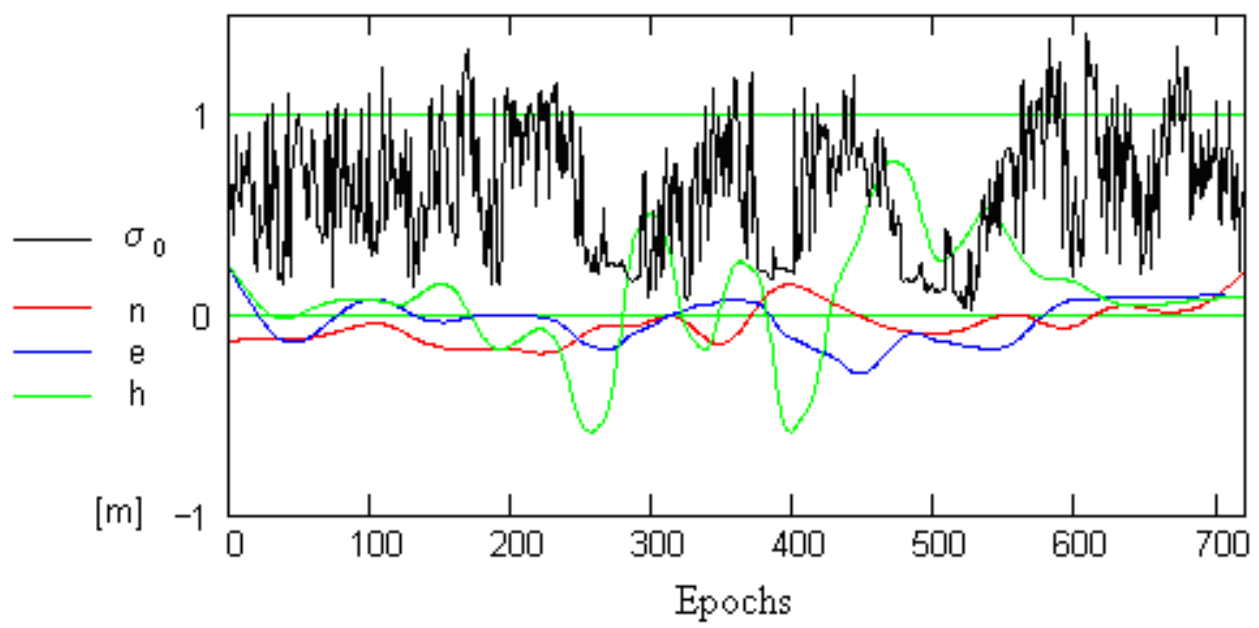

Fig. 7. Smoothed 3D position in local coordinate system and values of variance factor in network code DGPS adjustment

Furthermore, results of 5-min sessions in DGPS (Table 1 and 2) are presented based on these positions and accuracy obtained from the VC matrix. For all 5-min sessions in the network code GPS positioning, only those epochs where the determined accuracy was below $10 \mathrm{~cm}$ and then averaged in the specific session $\left(\mathrm{m}_{\mathrm{X}}\right.$, mean; $\mathrm{m}_{\mathrm{Y} \text {, mean }} ; \mathrm{m}_{\mathrm{Y}}$, mean $)$ were chosen. Results are presented in Tables 1 for the station TARG and in Table 2 for the station KATO. Next, another averaging process of real errors was conducted $\left(\Delta \mathrm{X}_{\text {mean }}, \Delta \mathrm{Y}_{\text {mean }}, \Delta \mathrm{Z}_{\text {mean }}\right)$ for particular sessions, i.e. where the variance factor was in the range from 0.8 to 1.2 ; these values of scale factor were chosen empirically. It can be seen that estimated and real accuracy coincides for the variance factor from 0.8 to 1.2. For the station TARG, only in session 8 did none of the satisfied results fulfill the required threshold of accuracy, i.e. the variance factor was not within $0.8-1.2$ and the accuracy for every component $(\Delta \mathrm{X}, \Delta \mathrm{Y}, \Delta \mathrm{Z})$ was below $10 \mathrm{~cm}$. But there were three sessions (T008, K008, K009) where no $\mathrm{cm}$ accuracy was obtained. It was impossible due to bad code accuracy, possibly caused by non-systematic errors. But those sessions were also reliable when estimated in terms of real accuracy obtained from the VC matrix. It should be remained at this point that for static baseline estimation, bad ambiguity resolutions caused errors of meters or so in final positions, and additionally showing that statistical tests of baseline solutions were passed successfully. For land surveying and for many GIS applications, valid accuracy estimation is essential process and needed for all 
points as additional note because accuracy of determined positions reflects their destination tasks.

Table 1. Real errors vs. estimated errors in network DGPS positioning for the TARG station in 5-min sessions

\begin{tabular}{|r|r|c|c|c|c|c|c|}
\hline Session & $\begin{array}{l}\text { Number } \\
\text { of } \\
\text { solutions }\end{array}$ & $\Delta \mathrm{X}_{\text {mean }}$ & $\Delta \mathrm{Y}_{\text {mean }}$ & $\Delta \mathrm{Z}_{\text {mean }}$ & $\mathrm{m}_{\mathrm{X}, \text { mean }}$ & $\mathrm{m}_{\mathrm{Y}, \text { mean }}$ & $\mathrm{m}_{\mathrm{Z} \text {, mean }}$ \\
\hline T001 & 9 & 0,06 & $-0,04$ & $-0,09$ & 0,06 & 0,08 & 0,06 \\
\hline T002 & 7 & $-0,01$ & 0,04 & $-0,01$ & 0,04 & 0,03 & 0,09 \\
\hline T003 & 4 & 0,01 & $-0,04$ & $-0,02$ & 0,08 & 0,06 & 0,04 \\
\hline T004 & 3 & 0,02 & 0,04 & 0,02 & 0,02 & 0,03 & 0,01 \\
\hline T005 & 3 & $-0,01$ & $-0,01$ & $-0,03$ & 0,06 & 0,01 & 0,05 \\
\hline T006 & 7 & 0,01 & $-0,02$ & $-0,06$ & 0,05 & 0,04 & 0,06 \\
\hline T007 & 8 & 0,04 & 0,04 & $-0,03$ & 0,05 & 0,05 & 0,05 \\
\hline T008 & - & - & - & - & - & - & - \\
\hline T009 & 6 & 0,05 & 0,00 & $-0,04$ & 0,06 & 0,05 & 0,08 \\
\hline T010 & 6 & 0,03 & 0,02 & $-0,03$ & 0,07 & 0,04 & 0,04 \\
\hline T011 & 9 & 0,01 & $-0,01$ & 0,00 & 0,05 & 0,03 & 0,04 \\
\hline T012 & 8 & 0,02 & 0,02 & 0,04 & 0,07 & 0,06 & 0,10 \\
\hline
\end{tabular}

Table 2. Real errors vs. estimated errors in network DGPS positioning for the KATO station in 5-min sessions

\begin{tabular}{|r|r|c|c|c|c|c|c|}
\hline Session & $\begin{array}{l}\text { Number } \\
\text { of } \\
\text { solutions }\end{array}$ & $\Delta \mathrm{X}_{\text {mean }}$ & $\Delta \mathrm{Y}_{\text {mean }}$ & $\Delta \mathrm{Z}_{\text {mean }}$ & $\mathrm{m}_{\mathrm{X} \text {, mean }}$ & $\mathrm{m}_{\mathrm{Y} \text { mean }}$ & $\mathrm{m}_{\mathrm{Z} \text {, mean }}$ \\
\hline $\mathrm{K} 001$ & 9 & 0,07 & $-0,02$ & $-0,07$ & 0,06 & 0,08 & 0,08 \\
\hline $\mathrm{K} 002$ & 9 & 0,02 & 0,01 & $-0,02$ & 0,04 & 0,04 & 0,02 \\
\hline $\mathrm{K} 003$ & 5 & 0,06 & $-0,03$ & $-0,11$ & 0,05 & 0,05 & 0,05 \\
\hline $\mathrm{K} 004$ & 4 & 0,02 & 0,02 & $-0,08$ & 0,02 & 0,07 & 0,08 \\
\hline $\mathrm{K} 005$ & 2 & 0,02 & 0,02 & $-0,05$ & 0,02 & 0,11 & 0,03 \\
\hline $\mathrm{K} 006$ & 5 & 0,00 & 0,01 & $-0,02$ & 0,04 & 0,07 & 0,09 \\
\hline $\mathrm{K} 007$ & 4 & 0,01 & $-0,02$ & 0,03 & 0,04 & 0,01 & 0,07 \\
\hline $\mathrm{K} 008$ & - & - & - & - & - & - & - \\
\hline $\mathrm{K} 009$ & - & - & - & - & - & - & - \\
\hline $\mathrm{K} 010$ & 1 & 0,05 & $-0,02$ & $-0,04$ & 0,03 & 0,07 & 0,07 \\
\hline $\mathrm{K} 011$ & 8 & $-0,02$ & 0,04 & 0,06 & 0,05 & 0,05 & 0,07 \\
\hline $\mathrm{K} 012$ & 11 & $-0,05$ & 0,00 & 0,05 & 0,03 & 0,04 & 0,08 \\
\hline
\end{tabular}

Although for the sessions T008, K008, K009 in network DGPS positioning we did not obtain accuracy better than $10 \mathrm{~cm}$ in the least-squares adjustment, the LS adjustment results are obtained in global coordinates system XYZ. However, if the results were transformed from those sessions into the local topocentric coordinates system (Fig 8), it should be noted that the main errors are included in the height component, whereas the horizontal northing and easting coordinates are mostly below $20 \mathrm{~cm}$ from the true coordinates. It is very important to note due to the fact that most surveying works need 2D positioning; it is also valid for GIS and navigation expectations. 
a)

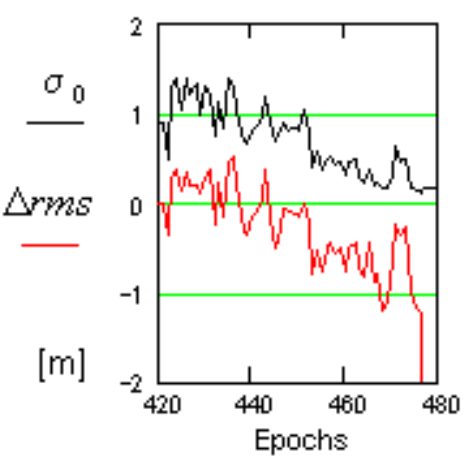

b)

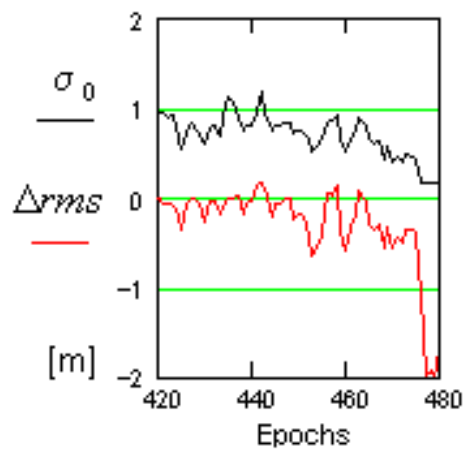

c)

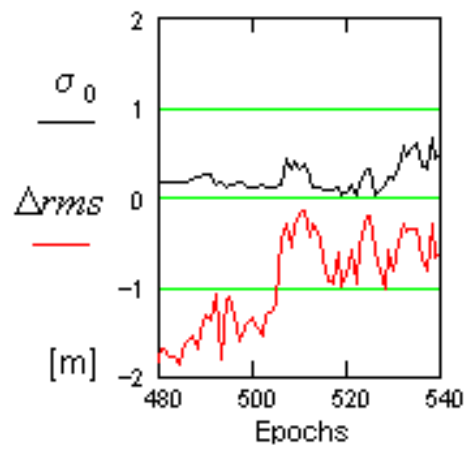

d)

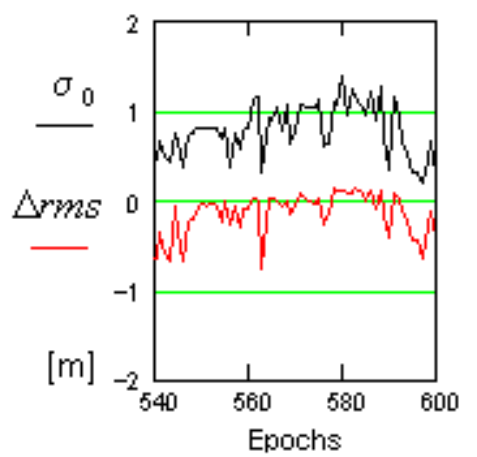

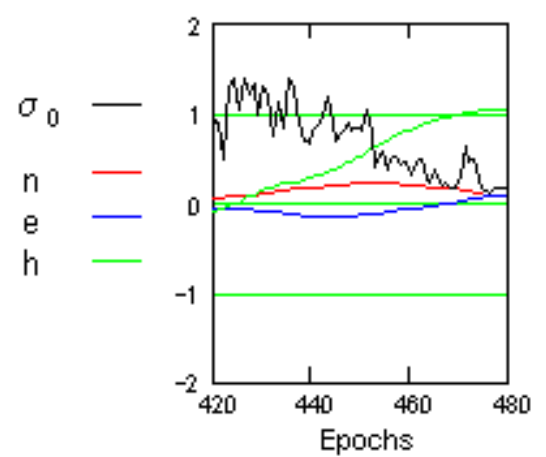
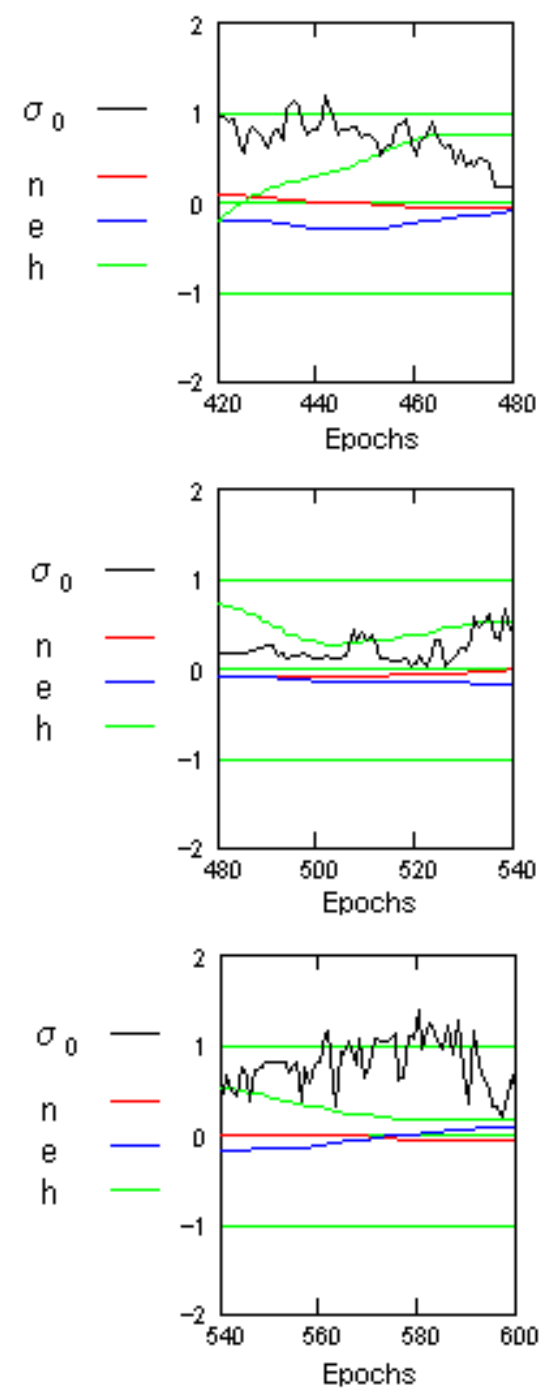

Fig. 8. Values of $\Delta r m s$, scale factor and coordinates for the following sessions of network code solutions: a) T008; b) K008; c) K009 and d) K010

Finally, in analyzing Fig. 8 one can see that the estimated accuracy by the VC matrix with the variance factor reflects the real accuracy of network code DGPS positioning. Although an accuracy better than $10 \mathrm{~cm}$ was not obtained, positions where the variance factor was between 0.8 and 1.2 had coordinates properly estimated. It is a very important issue from a surveying perspective in terms of validation of GPS positioning. For the session K009 (Fig. 8c) in network DGPS positioning there were no properly estimates, i.e. the variance factor was much more below a unity. However, analyzing this time of observation in static phase solutions we can find gross errors on some baselines although test statistics of the baselines processing passed successfully (see e.g. Fig $3 \mathrm{~b}$ and $3 \mathrm{e}$ ). Note that in code network positioning 
there were no misleading solutions. For the session K010 (Table 2) there was only one epoch fulfilled the accuracy threshold and that positions had also reliable accuracy estimated: $3 \mathrm{~cm}$, $7 \mathrm{~cm}$ and $7 \mathrm{~cm}$ for components $\mathrm{X}, \mathrm{Y}$ and $\mathrm{Z}$, respectively.

\section{SUMMARY and CONCLUSIONS}

Although currently network solutions rather concern RTK positioning, where $1-3 \mathrm{~cm}$ is expected in real time, but it should be remembered that RTK positioning needs very good satellite configuration and good quality of phase observation. In practice, however, there are many different tasks where access to the clear sky is limited (by trees, buildings etc.) and quick RTK positioning is not an easy process or even sometimes impossible. In such situations, valid RTK positions may be obtained after very long time sessions, which is why new methods of positioning are still very welcome and much more research is still required in order to fulfill practice requirements where different accuracy is expected - not only centimeter, but also decimeter.

This paper presents the approach of network code DGPS positioning where $\mathrm{cm}$ accuracy is achievable during 5-min observational sessions. The results were compared with the classical static carrier phase single baseline solutions using commercial software. When the static approach was tested there were different approaches of ambiguities solutions chosen. Although in static baselines all GPS data were used, the accuracy was not satisfied due to many gross errors in the final results. It means that static GPS baselines were not reliably calculated in such a short time of observation. Only for one baseline KLOB-TARG were the results within $10 \mathrm{~cm}$, in others, they were in the range of 1-3 meters.

For all 5-min sessions in network code GPS positioning, only those epochs where determined accuracy by VC matrix was below $10 \mathrm{~cm}$ were chosen and then averaged in the specific session. This obtained $\mathrm{cm}$ accuracy, i.e. the real accuracy was below $10 \mathrm{~cm}$ for those sessions. But there were three sessions which did not obtain $\mathrm{cm}$ accuracy. It was impossible due to bad code accuracy caused probably by non-systematic errors. But those sessions were also reliable when estimated in terms of real accuracy obtained from the VC matrix and a real horizontal accuracy below $20 \mathrm{~cm}$ was obtained after smoothing process; only the heights were much more inaccurate, i.e. there were epochs were errors amounted to 0.5-1 meter. At this point it should be clearly underlined that those real errors of heights were confirmed by the determined accuracy obtained from the VC matrix in the LS solution.

The proposed code network DGPS positioning is a reliable technique in terms of validation of GPS positioning and can be successfully used for many different GIS applications. For short observation times, this approach of network code DGPS seems to be very useful and $5-10 \mathrm{~cm}$ accuracy can be expected. This positioning can be very helpful tool in ambiguity resolution procedures as well as position validation in static positioning. In the future when many more satellites will exist by connecting with other independent satellite systems, it may be anticipated that code network positioning may become a reliable technique for GNSS positioning, especially in difficult observational conditions where only code measurements can be expected. 


\section{REFERENCES}

Ashtech. (1993). Ashtech XII GPPS, GPS Post-Processing System Manual, Software Version 5.0.00, USA

Ashtech and Spectra Precision Terrasat GmbH Germany. (1998). Ashtech Office Suite for Survey, User's Manual, USA.

Bakuła M. (2006a), An Approach of Network Code Differential GPS Positioning for Medium and Long Distances. Artificial Satellites, Journal of Planetary Geodesy, Vol. 41, No. 4, pp. 136-148.

Bakuła M. (2006b). Performance of Static Positioning for Medium Distances Based on Data from a Virtual Reference Station and ASG-PL Network, Artificial Satellites, Journal of Planetary Geodesy, Vol. 41, No. 1, pp. 33-42.

Bakuła M. Oszczak S. (2006). Experiences of RTK Positioning in Hard Observational Conditions During Nysa Kłodzka River Project, Vienna, Austria, Reports on Geodesy, No. 1(76), pp 71-79.

Bakuła M., Oszczak S., Pelc-Mieczkowska R., Suchocki M., Chrostowska M., Rudziński M. (2006). Analysis of Precision and Accuracy of GPS Measurements in Forest Conditions, Polskie Towarzystwo Informacji Przestrzennej, Rocznik GEOMATYKI, Tom IV, zeszyt 3, s. 23-32.

Grejner-Brzezińska D.A. Kashani I., Wielgosz P. (2005). On accuracy and reliability of instantaneous network RTK as a function of network geometry, station separation, and data processing strategies, GPS Solution, Vol. 9, N. 3, pp. 212-225.

Hofmann-Wellenhof B., Lichtenegger H., Collins J. (1997). Global Positioning System Theory and Practice, Fourth Edition, Springer-Verlag Wien New York.

Kleusberg A., Teunissen P.J.G. (eds) (1996). GPS For Geodesy. Springer, Berlin Heidelberg New York.

Lachapelle G., Alves P. (2002). Multiple Reference Station Approach: Overview and Current Research, Journal of Global Positioning Systems, Vol. 1, No 2, pp 133-136.

Landau H., Vollath U., Chen X. (2002). Virtual Reference Stations Systems, Journal of Global Positioning Systems, Vol. 1, No 2, pp 137-143.

Leick A. (1995). GPS Satellite Surveying, Second Edition, John Wiley \& Sons, INC.

Misra P., Enge P. (2001). Global Positioning System: Signals Measurement and Performance, Ganga-Jamuna Press, Massachusets.

Parkinson B., Spilker J.J. (eds). (1996). GPS Theory and Applications, vols 1 and 2, AIAA, Washington, DC.

Raquet, J. (1997). A New Approach to GPS Carrier Phase Ambiguity Resolution Using a Reference Receiver Network, Proceedings of National Technical Meeting, Santa Monica, January 14-16), The Institute of Navigation, Alexandria, VA, 357-366.

Strang G., Borre K. (1997). Linear Algebra, Geodesy and GPS, Wellesley-Combridge Press, USA.

Teunissen P. J. G., de Jonge P. J., Tiberius C. C. J. M. (1997). The least-squares ambiguity adjustment: its performance on short GPS baselines and short observation spans. Journal of Geodesy 71, pp. 589-602. 
Wanninger L. (1995). Improved Ambiguity Resolution by Regional Differential Modelling of the Ionosphere, Proc. of ION GPS 95, Palm Springs.

Wübbena G., Bagge A., Seeber G., Böder V., Hankemeier P. (1996). Reducing Distance Dependent Errors for Real-Time Precise DGPS Applications by Establishing Reference Station Networks, Proceedings of the International Technical Meeting, ION GPS-96, Kansas City, Missouri, pp. 1845-1852.

Received: 2008-01-28,

Reviewed: 2008-05-11, by M. Figurski,

Accepted: 2008-06-04. 